\title{
Moving Window Differential Evolution Independent Component Analysis-Based Operational Modal Analysis for Slow Linear Time-Varying Structures
}

\author{
Yongshuo Zong $\mathbb{D}^{1,2}$ Jinling Chen, ${ }^{1}$ Siyi Tao, ${ }^{1}$ Cheng Wang $\mathbb{D},{ }^{3}$ and Jianbing Xiahou $\mathbb{D}^{1}$ \\ ${ }^{1}$ School of Informatics, Xiamen University, Xiamen 361005, China \\ ${ }^{2}$ Department of Computer Science and Technology, Tongji University, Shanghai 201804, China \\ ${ }^{3}$ College of Computer Science and Technology, Huaqiao University, Xiamen, 361021, China
}

Correspondence should be addressed to Cheng Wang; wangcheng@hqu.edu.cn and Jianbing Xiahou; jbxiahou@xmu.edu.cn

Received 20 April 2020; Revised 8 October 2020; Accepted 19 October 2020; Published 23 November 2020

Academic Editor: Chenxi Huang

Copyright ( ) 2020 Yongshuo Zong et al. This is an open access article distributed under the Creative Commons Attribution License, which permits unrestricted use, distribution, and reproduction in any medium, provided the original work is properly cited.

\begin{abstract}
In order to identify time-varying transient modal parameters only from nonstationary vibration response measurement signals for slow linear time-varying (SLTV) structures which are weakly damped, a moving window differential evolution (DE) independent component analysis- (ICA-) based operational modal analysis (OMA) method is proposed in this paper. Firstly, in order to overcome the problems in traditional ICA-based OMA, such as easy to go into local optima and difficult-to-identify high-order modal parameters, we combine DE with ICA and propose a differential evolution independent component analysis- (DEICA-) based OMA method for linear time invariant (LTI) structures. Secondly, we combine the moving widow technique with DEICA and propose a moving window differential evolution independent component analysis- (MWDEICA-) based OMA method for SLTV structures. The MWDEICA-based OMA method has high global searching ability, robustness, and complexity of time and space. The modal identification results in a three-degree-of-freedom structure with slow time-varying mass show that this MWDEICA-based OMA method can identify transient time-varying modal parameters effectively only from nonstationary vibration response measurement signals and has better performances than moving window traditional ICA-based OMA.
\end{abstract}

\section{Introduction}

It is hoped that the engineering structure will have a high degree of self-adaptive or self-control ability, and it can automatically change its shape and performance to adapt to changes in environmental disturbances and new usage requirements as the environment or use functions change. Operational Modal Analysis (OMA) can estimate modal parameters without input data, which is not easy to obtain in large-scale engineering structures [1]. The modal parameters of a linear time-varying structure can reflect the overall dynamics of the structure [2].

Blind Source Separation (BSS) is a technique that recovers source signals from mixed signals without prior knowledge [3]. To cite a few, independent component analysis (ICA) [4, 5], second-order blind identification
(SOBI) [6], complexity pursuit $(\mathrm{CP})[7,8]$, and sparse component analysis (SCA) [9] are typical BSS methods. The ICA method requires that the source signals be independent of each other and the number of sensors is not less than the number of source signals [10]. Kerschen [11] is the first to recognize the mapping between the modal shapes and the columns of the mixing matrix in BSS and, then, apply the ICA method to OMA. Some methods have been proposed to improve the ICA's restrictions $[12,13]$. However, because its optimization method is easy to go into local optima, traditional ICA-based OMA has low robustness, and it is difficult to identify high-order modal parameters using that [14]. Proposed by Strorn and Price $[15,16]$ firstly, differential evolution (DE) has much higher robustness and stronger global searching ability compared to the classical optimization method, such as the Gradient descent [17], stochastic 
gradient descent [18], and quasi-Newton iteration method [19].

However, because the mixing matrix of BSS is a constant matrix, BSS-based algorithms can only be used for time invariant structures. In reality, most of the structural parameters change with time and are time-varying structures. The main research on the time-vary structure is based on the frozen-in coefficient method which is considering the timevarying system as time invariant over a short time. Ramnath defines the slow linearly time-varying (SLTV) structures as the change of the system coefficient is much slower than the change of the system solution [20]. The three main approaches of SLTV structures are time-frequency analysis [21], forgetting factor [22], and moving window [23, 24]. However, the time-frequency analysis method is not suitable for online identification. The performance of forgetting factor methods is closely related to prior knowledge, which is very troublesome.

Based on the moving window [24] and independent component analysis [15], this paper presents a moving window differential evolution independent component analysis- (MWDEICA-) based OMA method for weakly damped SLTV structures.

The primary contributions of this paper can be summarized as follows:

(1) In this paper, we propose a new DEICA-based OMA method to identify modal parameters only from stationary random response signals for LTI structures. Using DE algorithm to replace the traditional linear regression optimization algorithm to search the separation matrix, the DEICA-based OMA method has high global searching ability, robustness, and complexity of time and space. Compared with the traditional ICA method based on gradient optimization algorithm, OMA based on DEICA can identify higher-order modal parameters and has high recognition accuracy.

(2) A sliding window algorithm based on differential evolution independent analysis of sliding window is proposed by combining sliding window with the DEICA method. The instantaneous modal natural frequency and mode shape of the linear time-varying structure are identified by using the linear tracking characteristics of sliding window. This method can effectively identify the transient time-varying modal parameters only from the nonstationary random response measurement signals, which is better than the traditional moving window method. The modal identification method has better performance.

(3) We design a three-degree-of-freedom structure with slowly time-varying mass simulation to verify the validity and recognition accuracy of the MWDEICAbased OMA algorithm.

The remainder of this paper is organized as follows. In section 2, we describe MWDEICA in detail. The simulation verification is demonstrated in section 3 . Finally, we conclude this paper in section 4 .

\section{Theoretical Inference of Algorithm}

2.1. Differential Evolution Independent Component AnalysisBased OMA for the LTI Structure. The gradient-based optimization algorithm used by the traditional ICA is easy to fall into the local optimum. Therefore, there are problems such as low robustness and inability to recognize high-order modes when performing modal analysis on a continuum structure. Differential evolution algorithm has the ability to solve global optimization problems. The DEICA-based OMA method uses DE as an optimization algorithm which replaces the traditional linear regression method, and the core is to optimize the separation matrix $\mathbf{W}$. Therefore, DEICA-based OMA can identify high-order modes and has higher precision. The specific steps are as follows:

(1) The number of modes $m$ to be extracted, the number of structural finite element nodes $N$, the number of response data collection points $T$, and the response matrix of the structure $\mathbf{X}(t) \in \mathbb{R}^{N \times T}$ are determined, and then, $\quad \mathbf{Y}(t)=\mathbf{W} \mathbf{X}(t) . \quad \mathbf{Y}(t) \in \mathbb{R}^{m \times T} \quad$ and $\mathbf{W} \in \mathbb{R}^{m \times N}$.

(2) The steps of the differential evolution algorithm are adjusted. Since $\vec{w}_{r}(r=1,2, \ldots, m)$ needs to be extracted one by one, each individual $\vec{p}_{i}$ in the population is a $1 \times N$ vector, which is stored in the population matrix $\mathbf{P} . \mathbf{P}=\left(\vec{p}_{1}, \vec{p}_{2}, \ldots, \vec{p}_{i}, \ldots, \vec{p}_{\mathrm{NP}}\right)^{T}$ $\in \mathbb{R}^{\mathrm{NP} \times N} \quad$ and $\quad \vec{p}_{i}=\left(p_{i}(1), p_{i}(2), \ldots, p_{i}(j), \ldots, p_{i}\right.$ $(N))^{T}, j=1,2, \ldots, N ; i=1,2, \ldots, \mathrm{NP}$.

(3) $\vec{p}_{i}$ is zero-centered by $\vec{p}_{i}=\vec{p}_{i}-\mathbf{P P}^{T} \vec{p}_{i}$, $\vec{p}_{i}=\left(\vec{p}_{i} /\left|\vec{p}_{i}\right|\right)$ and starts loop. The kurtosis is used as a function, which is $\operatorname{kurt}\left(\vec{y}_{i}\right)=E\left\{\left(\vec{y}_{i}\right)^{4}\right\}-3(E$ $\left.\left\{\left(\vec{y}_{i}\right)^{2}\right\}\right)^{2}, \vec{y}_{i}=\vec{p}_{i} \mathbf{X}(t)$. Each offspring of results is also zero-centered. Convergence condition of the algorithm: the maximum number of iterations (4000 times) is exceeded or $\left|\vec{p}_{\text {best }}^{(g) T} \cdot \vec{p}_{\text {best }}^{(g+1)}-1\right|<$ $10^{-9}$. The optimal solution is $\vec{p}_{\text {best }}$, and let $\vec{w}_{r}=$ $\vec{p}_{\text {best }}$.

(4) $r=r+1$; return to step (2) until $r>m$.

The flow diagram is shown in Figure 1.

2.2. Nonstationary Response Signals Decomposition in the Modal Coordinate and MWDEICA-Based OMA for SLTV. Based on the "time-freezing" theory [24], the frozen-in coefficient method, the "short time invariant," and "quasistationary" assumptions, the nonstationary random response signals of the SLTV structures can be approximated as the stationary random response time series of LTI structures in short time interval. Figure 2 shows the length selection of moving window.

The choice of moving window length is fixed (the length is $L$ ), and the displacement responses $\mathbf{X}_{L}^{(i)} \in \mathbb{R}^{N \times L}$ can be decomposed by the modal expansion:

$$
\mathbf{X}_{L}^{(i)} \approx \boldsymbol{\Phi}_{L}^{(i)} \mathbf{Q}_{L}^{(i)}, \quad i=1,2, \ldots, T+1-L,
$$




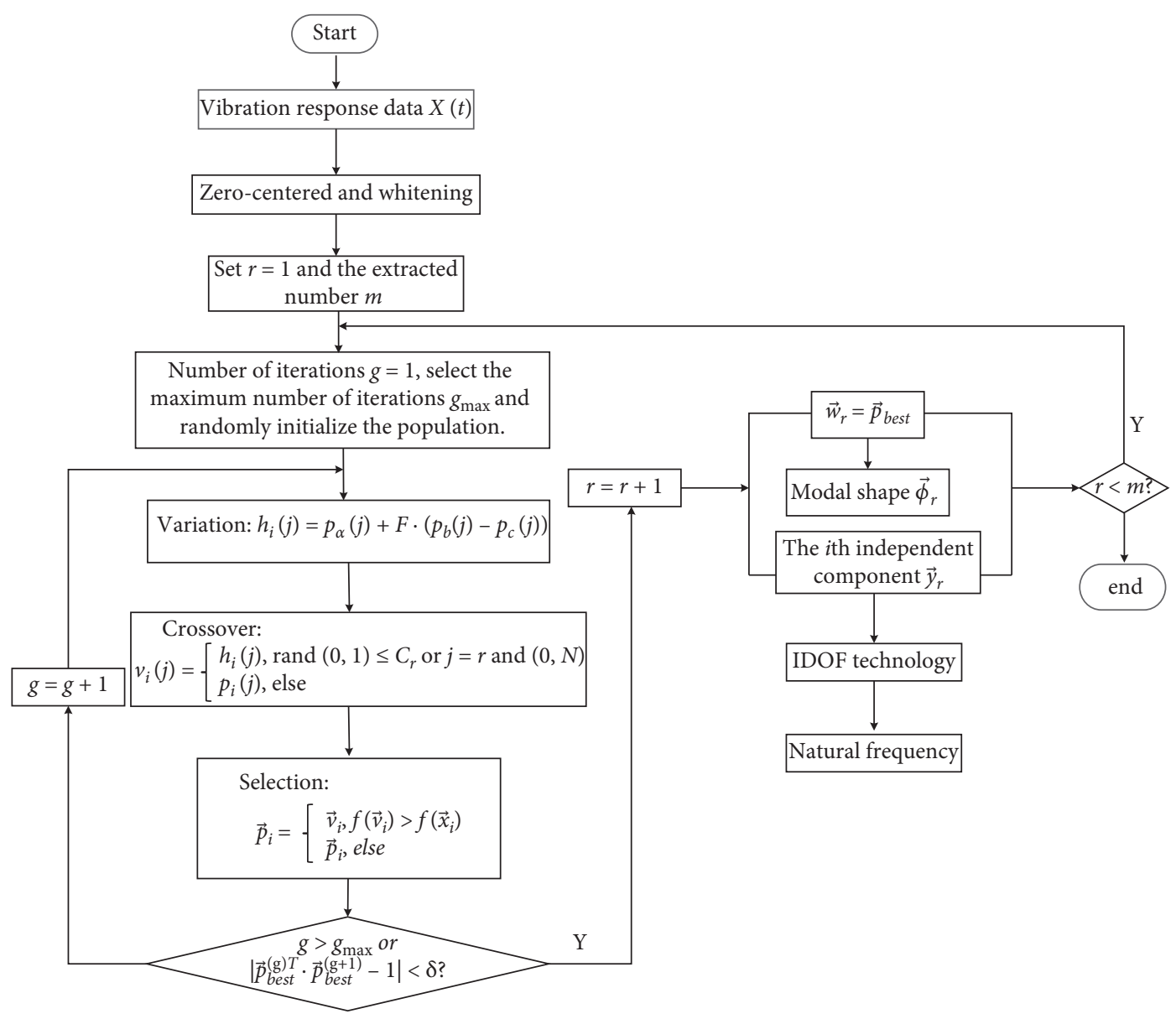

FIgUre 1: The flow diagram of DEICA-based OMA for the LTI structure.

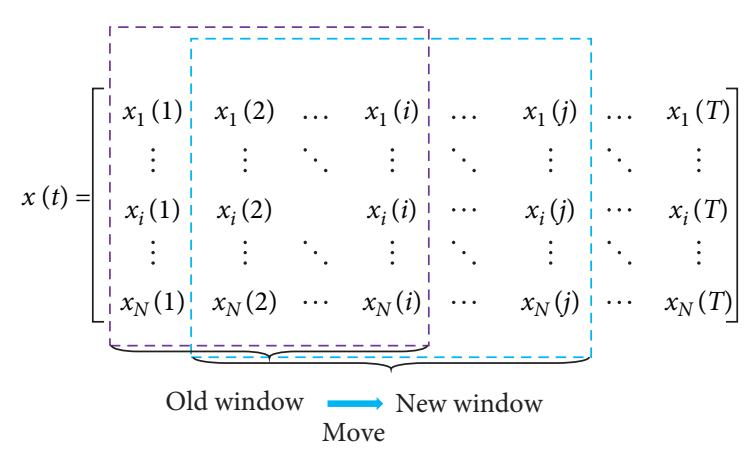

Figure 2: The description of fixed length moving window.

where $\Phi_{L}^{(i)}=\left[\vec{\phi}_{1}^{(i)}, \ldots, \vec{\phi}_{k^{\prime}}^{(i)}, \ldots, \vec{\phi}_{N}^{(i)}\right] \in \mathbb{R}^{N \times N}$ is the modal shapes matrix and $\mathbf{Q}_{L}^{(i)}=\left[\vec{q}_{1}^{(i)}, \ldots, \vec{q}_{k^{\prime}}^{(i)}, \ldots, \vec{q}_{N}^{(i)}\right] \in \mathbb{R}^{N \times L}$ is the modal coordinate response matrix. When the order of modal natural frequencies $f_{k^{\prime}}^{i}$ is not equal, the modal coordinate response vectors $\vec{q}_{k^{\prime}}^{(i)}$ are independent of each other.

From equation (1), we can see that $\Phi_{L}^{(i)}$ and $\mathbf{Q}_{L}^{(i)}$ represent, respectively, the LTV structure's statistical average modal shapes and modal response over the window time $L$. When time reaches $t_{i+(L-1) / 2}$, the predicted modal shape vector $\vec{\phi}_{k^{\prime}}(i+(L-1) / 2)$ approximates to $\vec{\phi}_{k^{\prime}}^{(i)}$. Similarly, after using the single-degree-of-freedom (DOF) technique, the nature frequency $f_{k^{\prime}}(i+(L-1) / 2)$ of $k^{\prime}$ th order at time $t_{i+(L-1) / 2}$ can also be replaced by $f_{k^{\prime}}^{(i)}$.

In view of equation (1), there is a one-to-one relationship between the modal response matrix $\Phi_{L}^{(i)}$ of weakly damped structures and the linear transformational matrix $\mathbf{W}_{L}^{(i)}$ in MWICA and a one-to-one mapping between the independent components $\mathbf{Y}_{L}^{(i)}$ of MWICA and the modal response matrix $\mathbf{Q}_{L}^{(i)}$ in equation (1). Through ICA decomposition, the uniqueness, certainty, and existence of the MWDEICA-based OMA method can be proved and shown in Figure 3.

2.3. Theoretical Analysis and Comparison between Traditional ICA and DEICA. As an optimization solution method, DE has much higher robustness and stronger global searching ability compared to the classical optimization method such as gradient descent, random gradient descent, and Newton iteration. Table 1 shows the characteristics of ICA and DEICA.

2.4. Application Scopes of the Proposed Method. The application scope of the OMA method based on moving window and DEICA is as follows: 


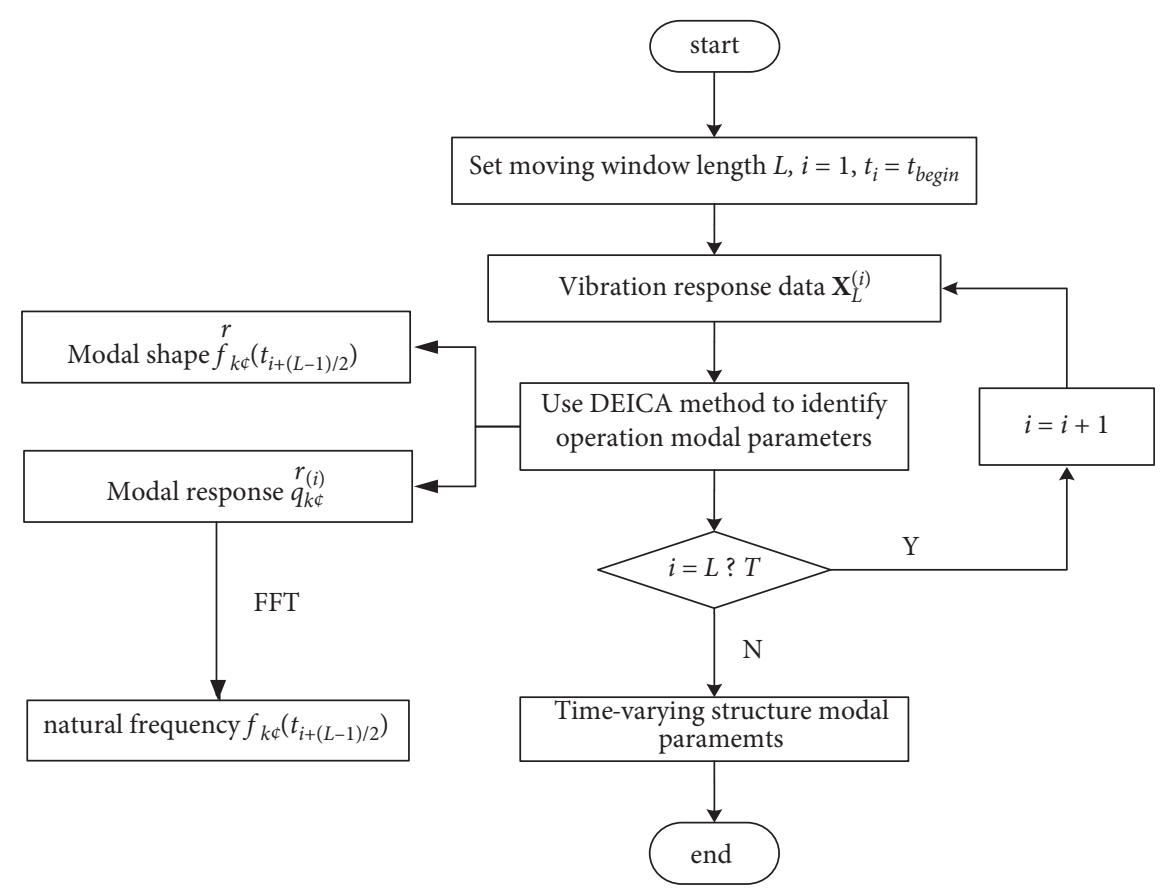

FIgURE 3: Process of MWDEICA-based OMA algorithm for SLTV structures.

TABLE 1: Theoretical analysis and comparison between traditional ICA and DEICA.

\begin{tabular}{lcc}
\hline & Traditional ICA & DEICA \\
\hline Fall into local optimum & Easy & Not easy \\
Global searching ability & Low & High \\
Robustness & Low & High \\
Complexity of time and space & Low & High \\
\hline
\end{tabular}

(1) The system should be a slow linear time-varying (SLTV) structure which is weakly damped.

(2) Because of the limitation of blind source separation and independent component analysis, the number of vibration response sensors should be greater than or equal to the identification order of identifiable operational modal parameters.

(3) Because of the limitation of sampling theorem, the sampling frequency of the vibration response signal should be greater than or equal to 2 times of the natural frequency of the identifiable modal natural frequency.

(4) Every order of modal shape is with different amplitude. In case of the ICA method, the energy of separation matrix is not unique, and independent component also loses amplitude information. Unlike the principal component analysis method [25], the ICA method cannot get the contribution ratio information of each modal. Modal shape is a relative quantity rather than an absolute value. So, in order to compare the modal shape with the real modal shape, the separation matrix and modal shape identified by the ICA method should be normalized.
(5) The order of modal parameter identified by ICA is uncertain. The modal identified by the ICA method is not in accordance with the order from small to large. In fact, the first separated output source and vector are the ones whose independence is the strongest rather than the first-order modal parameter. Therefore, in order to compare the natural frequencies with the real natural frequencies, the modal parameters identified by ICA need to be reordered by the modal frequencies.

\section{Simulation Identification}

3.1. Simulation Dataset of a Three-Degree-of-Freedom Structure with Slow Time-Varying Mass. To confirm MWICA's availability and identification accuracy, we have designed a simulation of an LTV three-DOF spring oscillator system in MATLAB/Simulink. This model is shown in Figure 4. The initial conditions for the three modal displacements and velocities are zero. The stiffness matrix is set to $k_{1}=1000(\mathrm{~N} / \mathrm{m}), \quad k_{2}=1000(\mathrm{~N} / \mathrm{m}), \quad$ and $\quad k_{3}=$ $1000(\mathrm{~N} / \mathrm{m})$, and the damping matrix is set to $c_{1}=$ $0.01(\mathrm{~N} \cdot \mathrm{s} / \mathrm{m}), c_{2}=0.01(\mathrm{~N} \cdot \mathrm{s} / \mathrm{m})$, and $c_{3}=0.01(\mathrm{~N} \cdot \mathrm{s} / \mathrm{m})$. The external force $F(t)=\left[\begin{array}{lll}F_{1}(t) & 0 & 0\end{array}\right]^{T}$ is white noise excitation with zero mean and unit variance. The mass matrix is set to $m_{2}=1 \mathrm{~kg}$ and $m_{3}=1 \mathrm{~kg}$, and $m_{1}$ is a timevarying parameter as follows:

$$
m_{1}(t)= \begin{cases}1, & 0 \leq t \leq 50, \\ e^{-0.0005(t-50)}, & 50<t \leq 2000 .\end{cases}
$$

This kinetic equation becomes 


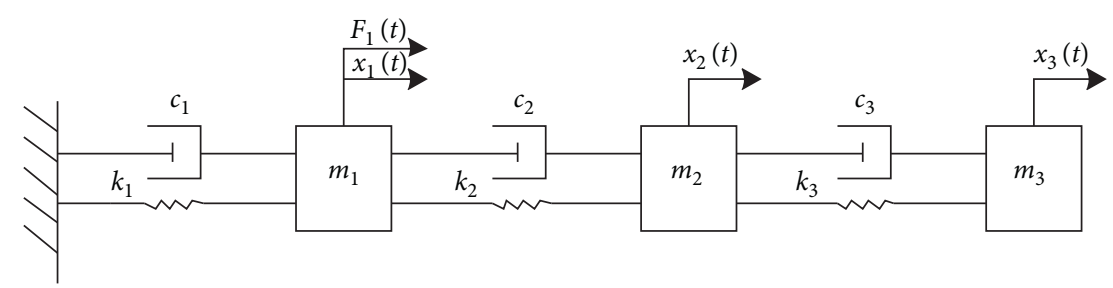

FIGURE 4: Linear time-varying three-degree-of-freedom spring vibrator model.

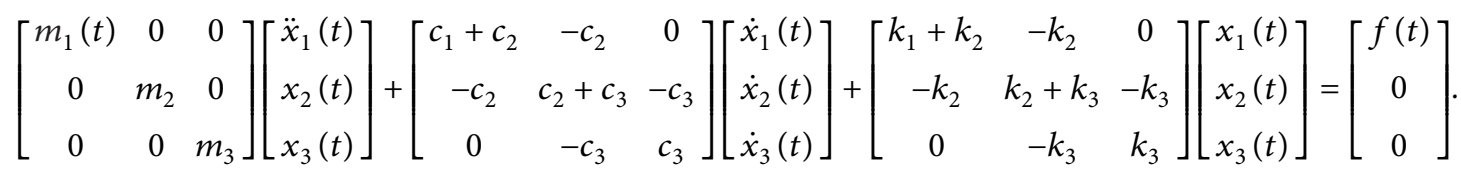

In the Simulink model, we use the Runge-Kutta algorithm to obtain responses of $40 \mathrm{~Hz}$ sampling frequency and $2000 \mathrm{~s}$ recorded time [23]. In the time domain, $2.0 \%$ level Gauss measurement noise disturbances are added to displacement response signals.
3.2. Simulation Parameters Setting. According to the third theoretical frequency $12.2951 \mathrm{~Hz}$ at time $1987.225 \mathrm{~s}$ in Table 2, the sampling frequency $f$ is set to $40 \mathrm{~Hz}$ in simulation of the SLTV structure. The window length is set to $L=1024$ in this simulation, and then, the frequency resolution of FFT $\Delta f=0.04 \mathrm{~Hz}$.

$$
\begin{aligned}
& \Delta f_{L}(1) \triangleq \frac{L}{f} \times \frac{f_{\text {end }}(1)-f_{\text {begin }}(1)}{t_{\text {end }}-t_{\text {begin }}}=\frac{1024}{40} \times \frac{2.31-2.24}{2000-50} \approx 9.25 \times 10^{-4} \mathrm{~Hz}, \\
& \Delta f_{L}(2) \triangleq \frac{L}{f} \times \frac{f_{\text {end }}(2)-f_{\text {begin }}(2)}{t_{\text {end }}-t_{\text {begin }}}=\frac{1024}{40} \times \frac{7.28-6.28}{2000-50} \approx 0.0131 \mathrm{~Hz}, \\
& \Delta f_{L}(3) \triangleq \frac{L}{f} \times \frac{f_{\text {end }}(3)-f_{\text {begin }}(3)}{t_{\text {end }}-t_{\text {begin }}}=\frac{1024}{40} \times \frac{12.33-9.07}{2000-50} \approx 0.0428 \mathrm{~Hz} .
\end{aligned}
$$

This objective function of ICA algorithm in this paper is the measurement of maximization non-Gaussian (kurtosis). The optimized method of class ICA algorithm is the quasiNewton iteration method. The ICA method is used multiple times simultaneously, and the maximum iterations time is 4000. The modal parameters can be identified most of the time in the case of 60 runs.

The parameter setting of DE: the individual number is 80; maximum iterations time is 4000 ; and proportionality factor $F$ and cross probability $C_{r}$ are random transformation each loop repeats.

3.3. Modal Assurance Criterion. To determine the accuracy of the modal shapes, the modal assurance criterion (MAC) is introduced to verify the operational modal identification results. The MAC is given by [26]

$$
\operatorname{MAC}_{\vec{\varphi}_{i}, \vec{\varphi}_{j}}=\frac{\left(\vec{\varphi}_{i}^{T} \vec{\varphi}_{j}\right)^{2}}{\left(\vec{\varphi}_{i}^{T} \vec{\varphi}_{j}\right)\left(\vec{\varphi}_{i}^{T} \vec{\varphi}_{j}\right)}
$$

where $\vec{\varphi}_{i}$ is the $i^{\text {th }}$ identified modal shape, $\vec{\varphi}_{\dot{q}}$ is the $j^{\text {th }}$ theoretical modal shape, and $\vec{\varphi}_{i}^{T}$ and $\vec{\varphi}_{j}$ are the transpositions of $\vec{\varphi}_{i}$ and $\vec{\varphi}_{j}$, respectively. The MAC values range between 0 (no coincidence) and 1 (complete coincidence). However, MAC only reveals information about the direction and shape of the modal results and does not contain any information about the modal amplitudes. The MAC value is always 1 if the two vectors are proportional.

\subsection{Transient Operational Modal Parameters Identification} Results. The two ICA algorithms were repeated 60 times, respectively. For the modal natural frequency, Figure 5 shows the theoretical transient natural frequencies and the identified natural frequencies by class MWICA and MWDEICA for the SLTV structure.

Figure 5 shows that certain modal parameters are not identified by class MWICA and MWDEICA at any time. In MWICA, the percentage of modal parameters of unidentified windows is more than $0.2 \%$ in the average of 60 trials.

We choose two moments $t=50.25 \mathrm{~s}$ and $t=1350.00 \mathrm{~s}$ to compare the theoretical modal shapes with the identified modal shapes. The recognition value of the frequency is similar in each simulation, while the modal shape recognition result fluctuates. We select the best value in 60 times. 
TABLE 2: The comparison of transient natural frequencies at moments $50.025 \mathrm{~s}$ and $1987.225 \mathrm{~s}$.

\begin{tabular}{lccc}
\hline Modal order & $50.025 \mathrm{~s}(\mathrm{~Hz})$ & $1987.225 \mathrm{~s}(\mathrm{~Hz})$ & Change of transient frequency $(\%)$ \\
\hline 1 & 2.24 & 2.31 & 3.173 \\
2 & 6.28 & 7.27 & 13.73 \\
3 & 9.07 & 12.30 & 26.24 \\
\hline
\end{tabular}

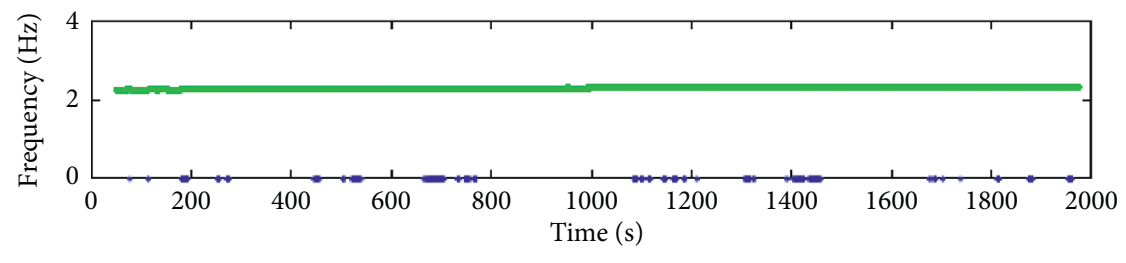

- Real frequency

+ Frequency indentified by MWICA

* Frequency indentified by MWDEICA

(a)

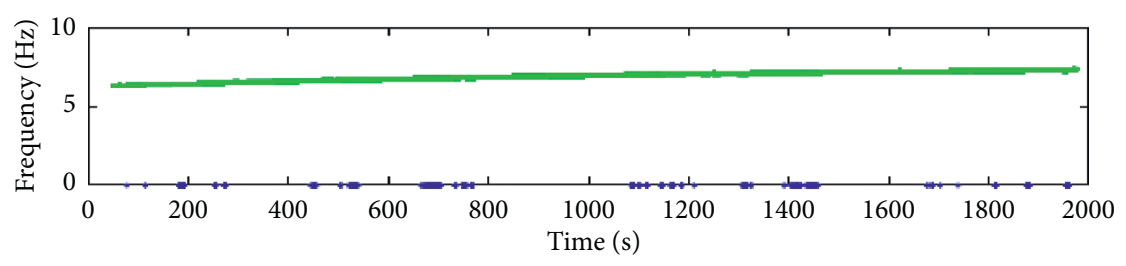

Real frequency

+ Frequency indentified by MWICA

* Frequency indentified by MWDEICA

(b)

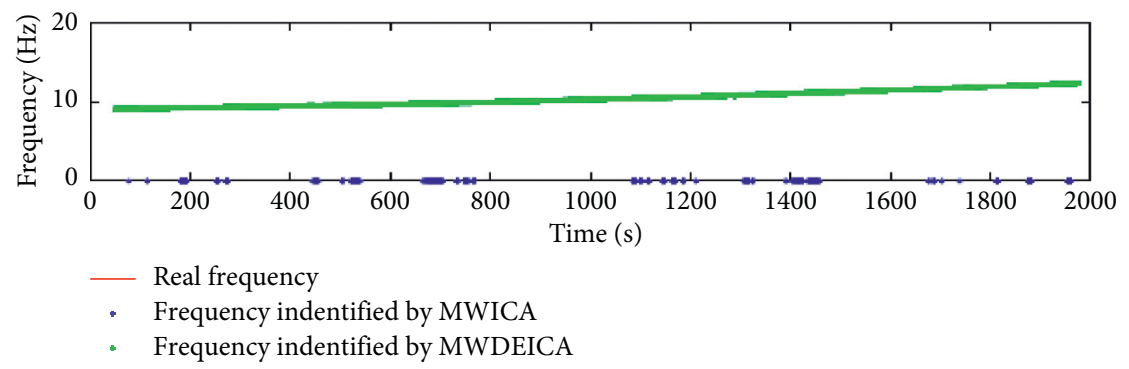

(c)

Figure 5: Comparison between the identified frequencies and theoretical frequencies. (a) Comparison of first-order modal frequencies, (b) Comparison of second-order modal frequencies, (c) Comparison of third-order modal frequencies.

\subsection{Results Analysis}

(1) Figures 5 and 6 and Tables 3 and 4 show that using only nonstationary random response signals, the MWICA-based and MWDEICAbased OMA method can well identify the transient natural frequency and modal shape of the SLTV structure.

(2) Figures 5, and 6 show that, in the case of adding $2.0 \%$ level Gauss measurement noise disturbances to displacement response signals, compared with the MWDEICA-based OMA method, the MVDEICA method can identify more modal parameters of windows, and the class MWICA-based OMA method has low robustness and it is difficult to identify modal parameters at some moment because its optimization method is easy to go into local optima [16]. For MWDEICA-based OMA, the recognition results are unstable.

(3) Table 5 shows that the MWDEICA-based OMA method has much higher time and space requirements than the MWICA-based OMA method. 


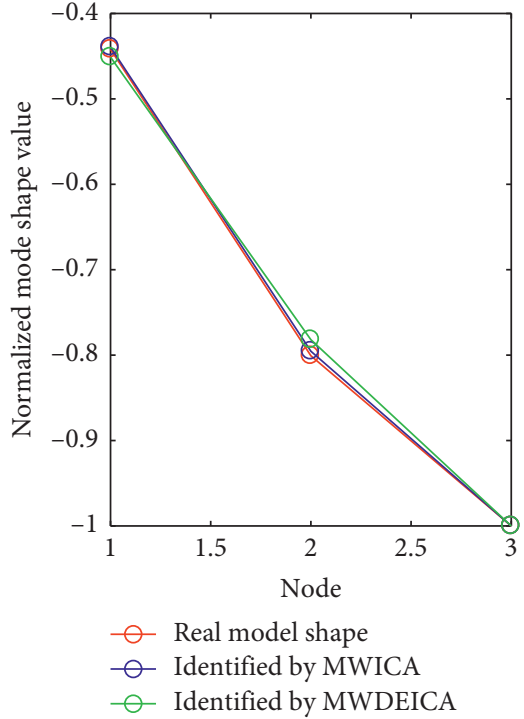

(a)

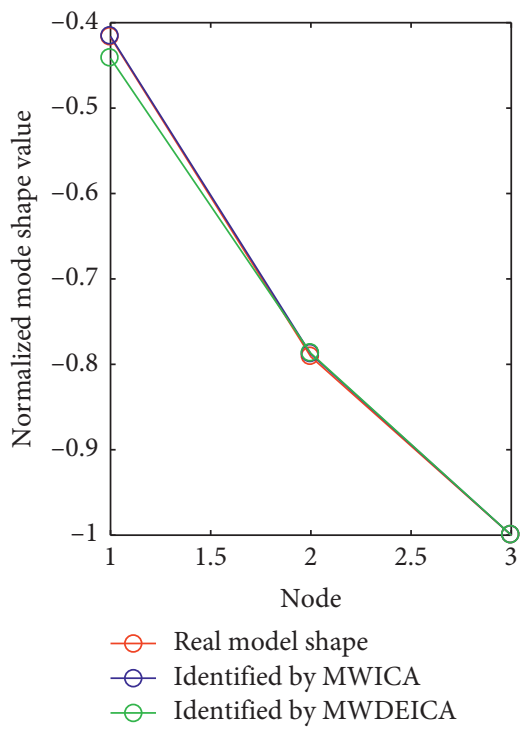

(d)

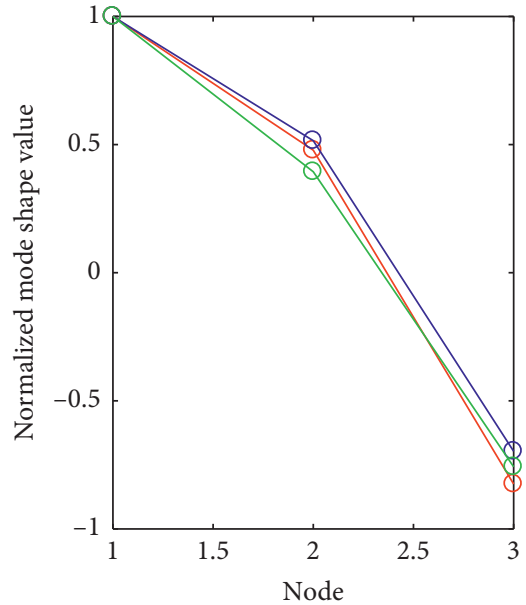

$\odot$ Real model shape

- Identified by MWICA

$\ominus$ Identified by MWDEICA

(b)

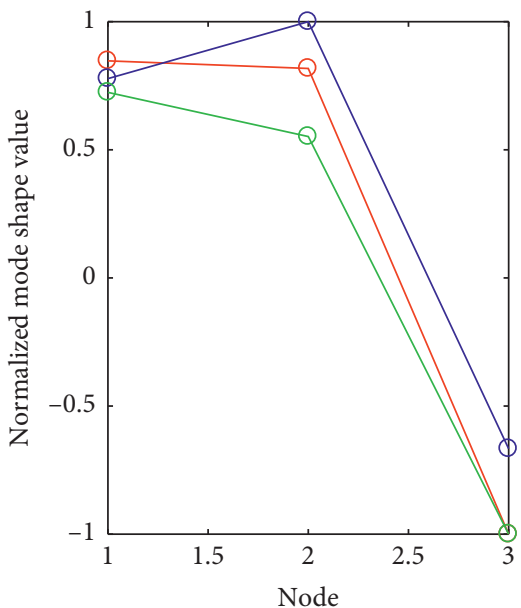

$\bigcirc$ Real model shape

- Identified by MWICA

$\_$Identified by MWDEICA

(e)

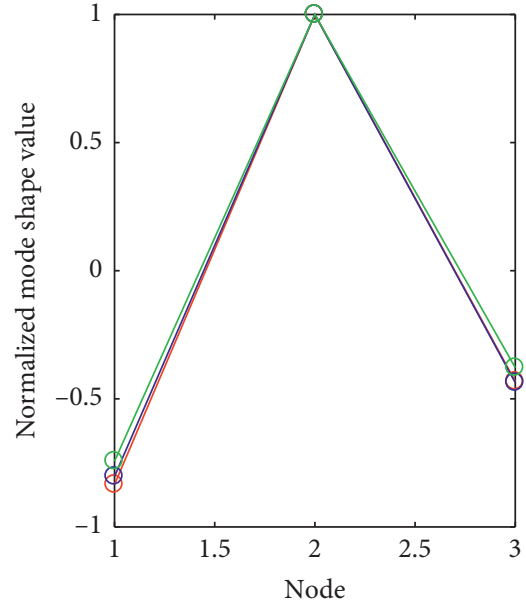

$\bigcirc$ Real model shape

- Identified by MWICA

- Identified by MWDEICA

(c)

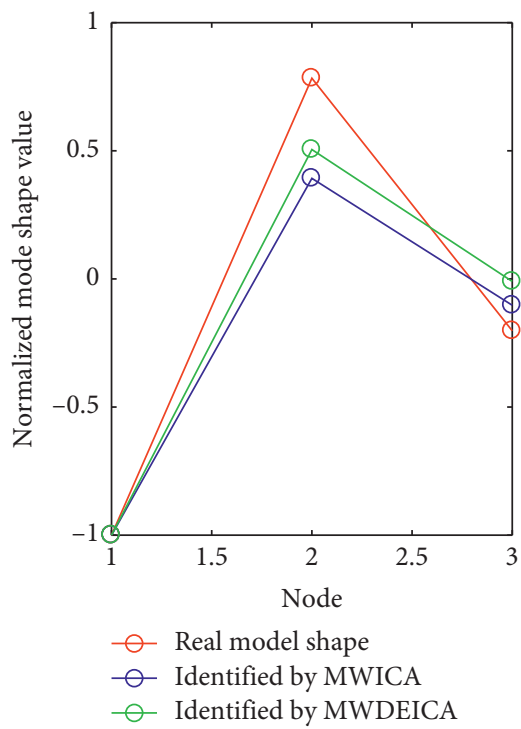

(f)

FIGURE 6: Comparison of the modal shape identified by MWICA and MWDEICA to the real modal shape at two moments. (a) When $t=50.25 \mathrm{~s}$, the $1^{\text {st }}$ modal shape, (b) when $t=50.25 \mathrm{~s}$, the $2^{\text {nd }}$ modal shape, (c) when $t=50.25 \mathrm{~s}$, the $3^{\text {rd }}$ modal shape, (d) when $t=1350 \mathrm{~s}$ the $1^{\text {st }}$ modal shape, (e) when $t=1350 \mathrm{~s}$ the $2^{\text {nd }}$ modal shape, and (f) when $t=1350 \mathrm{~s}$ the $3^{\text {rd }}$ modal shape.

TABLE 3: The MAC values comparison at time $50.25 \mathrm{~s}$.

\begin{tabular}{lcccc}
\hline Theory modal shape no. & $\begin{array}{c}\text { MWICA } \\
\text { identification order }\end{array}$ & $\begin{array}{c}\text { MWDEICA } \\
\text { identification order }\end{array}$ & $\begin{array}{c}\text { MAC identified } \\
\text { by MWICA }\end{array}$ & $\begin{array}{c}\text { MAC identified by } \\
\text { MWDEICA }\end{array}$ \\
\hline 1 & 2 & 1 & 1.0000 & 0.9998 \\
3 & 1 & 3 & 0.9922 & 0.9981 \\
2 & 3 & 2 & 0.9999 & 0.9979 \\
\hline
\end{tabular}

TABLE 4: The MAC values comparison at time $1350 \mathrm{~s}$.

\begin{tabular}{lcccc}
\hline Theory modal shape no. & $\begin{array}{c}\text { MWICA } \\
\text { identification order }\end{array}$ & $\begin{array}{c}\text { MWDEICA } \\
\text { identification order }\end{array}$ & $\begin{array}{c}\text { MAC identified } \\
\text { by MWICA }\end{array}$ & $\begin{array}{c}\text { MAC identified by } \\
\text { MWDEICA }\end{array}$ \\
\hline 1 & 1 & 1 & 1.0000 & 0.9998 \\
2 & 3 & 2 & 0.9419 & 0.9734 \\
3 & 2 & 3 & 0.9076 & 0.9326 \\
\hline
\end{tabular}


TABLE 5: Time and space requirements of different methods.

\begin{tabular}{lcc}
\hline Method & $\begin{array}{c}\text { Time spent } \\
\text { (second) }\end{array}$ & $\begin{array}{c}\text { Space requirement } \\
\text { (megabyte) }(\mathrm{MB})\end{array}$ \\
\hline $\begin{array}{l}\text { MWICA-based OMA } \\
\text { MWDEICA-based }\end{array}$ & 1700.3 & 103 \\
OMA & 6804.2 & 578 \\
\hline
\end{tabular}

\section{Conclusions}

In order to solve the problem that the traditional ICA method is easy to fall into local optimum and difficult to identify high-order modal parameters, combining moving window technology and differential evolution independent component analysis, this paper proposes a transient operational modal parameters identification method only from nonstationary vibration response measurement signal $s$ for slow linear time-varying structures which is weakly damped. Theoretical comparison and simulation identification results show that MWDEICA-based OMA can effectively identify time-varying modal parameters, while the parameters of some sample points are not recognized in MWICA.

Due to moving window technology and differential evolution algorithm, we are still unable to identify modal parameters in some time points. How to reduce the complexity of the algorithm and use MVDEICA to identify higher-order modals is the future work. It is also the future work to solve the problem and experimentally verify on more complex three-dimensional engineering structures.

\section{Data Availability}

The data used to support the findings of this study are available from the corresponding author upon request.

\section{Conflicts of Interest}

The authors declare that they have no conflicts of interest.

\section{Acknowledgments}

This work was financially supported by CERNET Innovation Project (grant no. NGII20160410).

\section{References}

[1] E. Reynders, "System identification methods for (operational) modal analysis: review and comparison," Archives of Computational Methods in Engineering, vol. 19, no. 1, pp. 51-124, 2012.

[2] K. Liu and M. R. Kujath, "Adaptation of the concept of modal analysis to time-varying structures," Mechanical Systems and Signal Processing, vol. 13, no. 3, pp. 413-422, 1999.

[3] W. Guan, L. L. Dong, J. M. Zhou, Y. Han, and J. Zhou, "Datadriven methods for operational modal parameters identification: a comparison and application," Measurement, vol. 132, pp. 238-251, 2019.

[4] J. Hang and Z. Yi, "Operational modal identification technique based on independent component analysis," in Proceedings of the 2011 International Conference on Electric
Technology and Civil Engineering (ICETCE), pp. 2430-2433, Lushan, China, April 2011.

[5] S. Chauhan, R. Martell, R. J. Allemang, and D. L. Brown, "Application of independent component analysis and blind source separation techniques to operational modal analysis," in Proceedings of the 25th IMAC, Orlando, FL, USA, February 2007.

[6] C. Rainieri, "Perspectives of second-order blind identification for operational modal analysis of civil structures," Shock and Vibration, vol. 2014, Article ID 845106, 2014.

[7] M. A. Eitner, B. G. Miller, J. Sirohi, and C. E. Tinney, "Operational modal analysis of a thin-walled rocket nozzle using phase-based image processing and complexity pursuit," in Rotating Machinery, Optical Methods \& Scanning LDV Methods, vol. 6, pp. 19-29, Springer, Cham, Switzerland, 2019.

[8] J. Antoni, R. Castiglione, and L. Garibaldi, "Interpretation and generalization of complexity pursuit for the blind separation of modal contributions," Mechanical Systems and Signal Processing, vol. 85, pp. 773-788, 2017.

[9] H. Zhou, K. Yu, Y. Chen, R. Zhao, and Y. Wu, "Output-only modal estimation using sparse component analysis and density-based clustering algorithm," Measurement, vol. 126, pp. 120-133, 2018.

[10] M. D. Spiridonakos, E. N. Chatzi, and B. Sudret, "Polynomial chaos expansion models for the monitoring of structures under operational variability," ASCE-ASME Journal of Risk and Uncertainty in Engineering Systems, Part A: Civil Engineering, vol. 2, no. 3, Article ID B4016003, 2016.

[11] G. Kerschen, F. Poncelet, and J.-C. Golinval, "Physical interpretation of independent component analysis in structural dynamics," Mechanical Systems and Signal Processing, vol. 21, no. 4, pp. 1561-1575, 2007.

[12] C. Wang, J. Wang, X. Lai et al., "Operation modal analysis following fast independent component analysis," International Journal of Applied Electromagnetics and Mechanics, vol. 52, no. 1-2, pp. 103-111, 2016.

[13] C. Wang, J. Wang, B. Zhong et al., "Negentropy and gradient iteration based fast independent component analysis for multiple random fault sources blind identification and separation," International Journal of Applied Electromagnetics and Mechanics, vol. 52, no. 1-2, pp. 711-719, 2016.

[14] J. Wang, C. Wang, T. Zhang, and B. Zhong, "Comparison of different independent component analysis algorithms for output-only modal analysis," Shock and Vibration, vol. 2016, pp. 1-25, 2016.

[15] R. Storn and K. Price, "Differential evolution-a simple and efficient heuristic for global optimization over continuous spaces," Journal of Global Optimization, vol. 11, no. 4, pp. 341-359, 1997.

[16] P. Civicioglu and E. Besdok, "A conceptual comparison of the cuckoo-search, particle swarm optimization, differential evolution and artificial bee colony algorithms," Artificial Intelligence Review, vol. 39, no. 4, pp. 315-346, 2013.

[17] C. Burges, T. Shaked, E. Renshaw et al., "Learning to rank using gradient descent," in Proceedings of the 22nd International Conference on Machine Learning (ICML-05), pp. 89-96, Bonn, Germany, August 2005.

[18] M. Zinkevich, M. Weimer, L. Li, and A. J. Smola, "Parallelized stochastic gradient descent," Advances in Neural Information Processing Systems, vol. 23, no. 23, pp. 2595-2603, 2010.

[19] D. F. Shanno, "Conditioning of Quasi-Newton methods for function minimization," Mathematics of Computation, vol. 24, no. 111, p. 647, 1970. 
[20] R. V. Ramnath, Multiple Scales Theory and Aerospace Applications, Multiple Scales Theory and Aerospace Applications, American Institute of Aeronautics and Astronautics, Reston, VA, USA, 2015.

[21] A. Klepka and T. Uh, "Identification of modal parameters of non-stationary systems with the use of wavelet based adaptive filtering," Mechanical Systems and Signal Processing, vol. 47, no. 1-2, pp. 21-34, 2014.

[22] W. Guan, C. Wang, D. S. Chen, X. Luo, and F. F. Su, "Recursive principal component analysis with forgetting factor for operational modal analysis of linear time-varying system," International Journal of Applied Electromagnetics and Mechanics, vol. 52, no. 3-4, pp. 999-1006, 2016.

[23] C. Wang, J. Wang, and T. Zhang, "Operational modal analysis for slow linear time-varying structures based on moving window second order blind identification," Signal Processing, vol. 133, pp. 169-186, 2017.

[24] C. Wang, H. Huang, J. Chen, W. Wei, and T. Wang, "An online and real-time adaptive operational modal parameter identification method based on fog computing in internet of things," International Journal of Distributed Sensor Networks, vol. 16, no. 1, pp. 1-12, 2020.

[25] C. Wang, J. Gou, J. Bai, and Y. Guirong, "Modal parameter identification with principal component analysis," Journal of Xi'an Jiaotong University, vol. 47, no. 11, pp. 97-104, 2013.

[26] J. Zou, K. Yu, and B. Yang, "Methods of time-varying structural parameter identification," Advances in Mechanics, vol. 30, no. 3, pp. 370-377, 2000. 